\title{
Discovery Of Lagrangian Equation For Fluid MECHANICS
}

\author{
Shuh Jing Ying \\ University of South FloridaTampa, Florida, USA
}

\begin{abstract}
This paper is intended to build a connection between dynamics and fluid mechanics. Since Lagrangian equations are very useful tools in dynamics now it is discovered that it can be also used in fluid mechanics, so connection can be built through this equation... Here, the lagrangian equation is derived from the momentum equation in fluid mechanics, and then the equation is applied to three different coordinates, Cartesian, cylindrical and spherical. Certainly the application of the equation is not limited to these three different coordinates. This is just for illustration. Many applications are expected.
\end{abstract}

\section{KEYWORDS}

Lagrangian equation, Dynamics and fluid mechanics are connected

\section{INTRODUCTION}

Fluid mechanics is my major in my graduate study. In my memory when I started to study the fluid mechanics, I learned a complete set of different equations in fluid mechanics. It seems not much related with dynamics. Here I hope that I can get some connections between dynamics and fluid mechanics. In dynamics, Lagrangian equations are very useful tools;many equations are developed with the use of Lagrangian equation. Now based on the momentum equation in fluid mechanics, I derived the Lagrangian equation, so I prove that Lagrangian equation can be also used in fluid mechanics. To simplify the derivation, I started the derivation for incompressible fluid, so a more general form of Lagrangian equation can be further developed. To illustrate the use of the equation, some examples are given. Those are especially in very familiar area, so people can immediately recognize that the equation is working. Momentum equations for inviscid incompressible fluid in Cartesian, cylindrical and spherical coordinates are chosen for the illustration.

\section{DERIVATION OF LAgRANGIAN EQUATION}

In dynamics, especially in the Chapter of Lagrange's equations, the position vector $\mathbf{r}$ is considered as a function of generalized coordinates $q_{i}$ and time $t$.

$\mathbf{r}=\mathbf{r}\left(\mathrm{q}_{\mathrm{i}}, \mathrm{t}\right)$

And velocity $\dot{\mathbf{r}}$ is a function of generalized coordinatesq $q_{i}$, generalized velocity $\dot{\mathrm{q}}_{i}$ and time $\mathrm{t}$.

$$
\dot{\mathbf{r}}=\dot{\mathbf{r}}\left(\mathrm{q}_{\mathrm{i}}, \dot{\mathrm{q}}_{\mathrm{i}}, \mathrm{t}\right)
$$

These notations will be used here in this paper. 
Mechanical Engineering: An International Journal (MEIJ), Vol. 4, No. 1/2/3/4, November 2017

In fluid mechanics, for incompressible fluid, when a portion of fluid is observed, following this portion of fluid, the momentum equation can be written as

$$
\rho \frac{\mathbf{D V}}{\mathrm{Dt}}-\mathbf{F}=0
$$

Where $\rho$ is density, $\frac{\mathbf{D}}{\mathbf{D} \mathbf{t}}$ is the substantial derivative, $\mathbf{V}$ is velocity and $\mathbf{F}$ is force per volume. Applying the virtual work concept, the equation becomes

$$
\left(\rho \frac{\mathrm{DV}}{\mathrm{Dt}}-\mathbf{F}\right) \cdot \mathrm{d} \mathrm{r}=0
$$

Since the virtual work is done at one instant of time, $\mathbf{r}$ is function of generalized coordinates only, so

$$
\left(\rho \frac{\mathbf{D V}}{\mathbf{D t}}-\mathbf{F}\right) \cdot \sum_{\mathrm{i}} \frac{\partial \mathbf{r}}{\partial \mathrm{q}_{\mathrm{i}}} \mathrm{dq}_{\mathrm{i}}=0
$$

Now equation (3) is to be considered by two parts, first part is

$$
\begin{aligned}
\rho \frac{\mathbf{D V}}{\mathbf{D t}} \cdot \frac{\partial \boldsymbol{r}}{\partial \mathrm{q}_{\mathrm{i}}} \mathrm{dq}_{\mathrm{i}} & =\rho\left[\left(\frac{\mathbf{D}}{\mathbf{D t}} \dot{\mathbf{r}}\right) \cdot \frac{\partial \mathbf{r}}{\partial \mathrm{q}_{\mathrm{i}}}\right] \mathrm{dq}_{\mathrm{i}} \\
& =\rho\left[\frac{\mathbf{D}}{\mathbf{D t}}\left(\dot{\mathbf{r}} \cdot \frac{\partial \mathbf{r}}{\partial \mathrm{q}_{\mathrm{i}}}\right)-\mathbf{r} \cdot \frac{\mathbf{D}}{\mathbf{D t}} \frac{\partial \mathbf{r}}{\partial \mathrm{q}_{\mathrm{i}}}\right] \mathrm{dq}_{\mathrm{i}} \\
& =\rho\left[\frac{\mathbf{D}}{\mathbf{D t}}\left(\dot{\mathbf{r}} \cdot \frac{\partial \mathbf{r}}{\partial \mathrm{q}_{\mathrm{i}}}\right)-\dot{\mathbf{r}} \cdot \frac{\partial \dot{\mathbf{r}}}{\partial \mathrm{q}_{\mathrm{i}}}\right] \mathrm{dq}_{\mathrm{i}}
\end{aligned}
$$

Since $\frac{\partial \mathbf{r}}{\partial \mathrm{q}_{\mathrm{i}}}=\frac{\partial \dot{\mathbf{r}}}{\partial \dot{\mathrm{q}}_{\mathrm{i}}}$ which is proved in Equation $(4-12)$ in Ying's Advanced Dynamics [1] and $\dot{\mathbf{r}}=\mathbf{V}$,

$$
\begin{aligned}
\text { L.H.S. } & =\rho\left[\frac{\mathrm{D}}{\mathrm{Dt}}\left(\frac{\partial}{\partial \dot{\mathrm{q}}_{\mathrm{i}}} \frac{\mathrm{v}^{2}}{2}\right)-\dot{\mathrm{r}} \cdot \frac{\partial \dot{\mathrm{r}}}{\partial \mathrm{q}_{\mathrm{i}}}\right] \mathrm{dq}_{\mathrm{i}} \\
& =\rho\left[\frac{\mathrm{D}}{\mathrm{Dt}}\left(\frac{\partial \mathrm{T}}{\partial \dot{\mathrm{q}}_{\mathrm{i}}}\right)-\frac{\partial \mathrm{T}}{\partial \mathrm{q}_{\mathrm{i}}}\right] \mathrm{dq}_{\mathrm{i}}
\end{aligned}
$$

where $\mathrm{T}=\frac{\mathrm{V}^{2}}{2}$ kinetic energy per unit mass. For the other term

$$
\mathbf{F} \cdot \frac{\partial \mathbf{r}}{\partial \mathrm{q}_{i}} \mathrm{dq}_{\mathrm{i}}=\mathrm{Q}_{\mathrm{i}} \mathrm{dq}_{\mathrm{i}}
$$

Where $Q_{i}$ is called generalized force $=\mathbf{F} \bullet \frac{\partial \mathbf{r}}{\partial q_{i}}$. Hence by combining equations (4) and (5) with (3), we Have 


$$
\sum_{\mathrm{i}}\left\{\rho\left[\frac{\mathrm{D}}{\mathrm{Dt}}\left(\frac{\partial \mathrm{T}}{\partial \dot{q}_{\mathrm{i}}}\right)-\frac{\partial \mathrm{T}}{\partial \mathrm{q}_{\mathrm{i}}}\right]-\mathrm{Q}_{\mathrm{i}}\right\} \mathrm{dq}_{\mathrm{i}}=0
$$

Because all the $\mathrm{q}_{i}$ areindependent, the coefficients must be zero. Therefore,

$$
\rho\left[\frac{\mathrm{D}}{\mathrm{Dt}}\left(\frac{\partial \mathrm{T}}{\partial \dot{\mathrm{q}}_{\mathrm{i}}}\right)-\frac{\partial \mathrm{T}}{\partial \mathrm{q}_{\mathrm{i}}}\right]=\mathrm{Q}_{\mathrm{i}}
$$

This is the Lagrangian equation for incompressible fluid in fluid mechanics.

\section{ApPlication}

To illustrate the use of the equation three different coordinates are chosen, Cartesian, cylindrical andspherical. Since they are familiar to everybody, they can be recognized easily.

A. Cartesian Coordinates

$$
\begin{aligned}
& \mathrm{d} \mathbf{r}=\mathbf{i} d x+\mathbf{j} d y+\mathbf{k} d z \\
& \dot{\mathbf{r}}=\mathbf{I} \dot{\mathrm{x}}+\mathbf{j} \dot{\mathrm{y}}+\mathbf{k} \dot{\mathrm{z}} \\
& \mathrm{T}=\frac{1}{2} \mathrm{~V}^{2}=\frac{1}{2}\left(\dot{\mathrm{x}}^{2}+\dot{\mathrm{y}}^{2}+\dot{\mathrm{z}}^{2}\right)
\end{aligned}
$$

Applying the Lagrangian equation

As $\mathrm{q}_{\mathrm{i}}=\mathrm{x}$, we have

$$
\begin{gathered}
\rho\left[\frac{\mathrm{D}}{\mathrm{D} t} \frac{\partial \mathrm{T}}{\partial \dot{\mathrm{x}}}-\frac{\partial \mathrm{T}}{\partial \mathrm{x}}\right]=\mathrm{Q}_{\mathrm{x}} \\
\rho \frac{\mathrm{D} \dot{\mathrm{D}}}{\mathrm{Dt}}=\mathrm{F}_{\mathrm{x}} \\
\rho\left(\frac{\partial \mathrm{u}}{\partial \mathrm{t}}+\mathrm{u} \frac{\partial \mathrm{u}}{\partial \mathrm{x}}+\mathrm{v} \frac{\partial \mathrm{u}}{\partial \mathrm{y}}+\mathrm{w} \frac{\partial \mathrm{u}}{\partial z}\right)=-\frac{\partial \mathrm{p}}{\partial \mathrm{x}}
\end{gathered}
$$

where $u=\dot{x}, v=\dot{y}, w=\dot{z}$ and $P$ is pressure.

Similarly for $\mathrm{q}_{\mathrm{i}}=\mathrm{y}$, we have

$$
\begin{gathered}
\rho \frac{\mathrm{Dy}}{\mathrm{Dt}}=\mathrm{F}_{\mathrm{y}} \\
\rho\left(\frac{\partial \mathrm{v}}{\partial \mathrm{t}}+\mathrm{u} \frac{\partial \mathrm{v}}{\partial \mathrm{x}}+\mathrm{v} \frac{\partial \mathrm{v}}{\partial \mathrm{y}}+\mathrm{w} \frac{\partial \mathrm{v}}{\partial \mathrm{z}}\right)=-\frac{\partial \mathrm{p}}{\partial \mathrm{y}}
\end{gathered}
$$

For $\mathrm{q}_{\mathrm{i}}=\mathrm{z}$, we have 
Mechanical Engineering: An International Journal (MEIJ), Vol. 4, No. 1/2/3/4, November 2017

$$
\begin{gathered}
\rho \frac{\mathrm{D} \dot{z}}{\mathrm{Dt}}=\mathrm{F}_{\mathrm{z}} \\
\rho\left(\frac{\partial \mathrm{w}}{\partial \mathrm{t}}+\mathrm{u} \frac{\partial \mathrm{w}}{\partial \mathrm{x}}+\mathrm{v} \frac{\partial \mathrm{w}}{\partial \mathrm{y}}+\mathrm{w} \frac{\partial \mathrm{w}}{\partial z}\right)=-\frac{\partial \mathrm{p}}{\partial z}
\end{gathered}
$$

\section{B. Cylindrical Coordinates}

$$
\begin{gathered}
\mathrm{d} \mathbf{r}=\mathbf{e}_{\mathrm{R}} \mathrm{d} \mathbf{R}+\mathbf{e}_{\varphi} \mathrm{Rd} \varphi+\mathbf{k} \mathrm{dz} \\
\mathrm{T}=\frac{1}{2} \mathrm{~V}^{2}=\frac{1}{2}\left[\dot{\mathrm{R}}^{2}+(\mathrm{R} \dot{\varphi})^{2}+\dot{z}^{2}\right]
\end{gathered}
$$

For $\mathrm{q}_{\mathrm{i}}=\mathrm{R}$, we have

$$
\begin{gathered}
\rho\left[\frac{\mathrm{D}}{\mathrm{Dt}} \frac{\partial \mathrm{T}}{\partial \dot{\mathrm{R}}}-\frac{\partial \mathrm{T}}{\partial \mathrm{R}}\right]=\mathrm{Q}_{\mathrm{R}} \\
\rho\left[\frac{\mathrm{D}}{\mathrm{Dt}} \dot{\mathrm{R}}-\mathrm{R} \dot{\varphi}^{2}\right]=\mathrm{Q}_{\mathrm{R}} \\
\rho\left[\frac{\mathrm{DV}}{\mathrm{Dt}}-\frac{\mathrm{V}_{\varphi}^{2}}{\mathrm{R}}\right]=\mathrm{F}_{\mathrm{R}}=-\frac{\partial \mathrm{P}}{\partial \mathrm{R}}
\end{gathered}
$$

For $\mathrm{q}_{\mathrm{i}}=\varphi$, we have

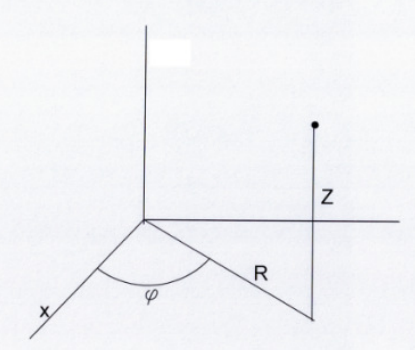

Cylindrical Coordinates

$$
\rho\left[\frac{\mathrm{D}}{\mathrm{Dt}}\left(\mathrm{R}^{2} \dot{\varphi}\right)\right]=\mathrm{Q}_{\varphi}=\mathrm{F}_{\varphi} \mathrm{R}
$$

Since $\frac{\partial \mathbf{r}}{\partial \varphi}=\mathbf{e}_{\varphi} \mathrm{R}$.

$$
\rho\left[\frac{\mathrm{D}}{\mathrm{Dt}}\left(\mathrm{RV}_{\varphi}\right)\right]=\rho \mathrm{R}\left[\frac{\mathrm{DV} \mathrm{V}_{\varphi}}{\mathrm{Dt}}+\frac{\mathrm{V}_{\mathrm{R}} \mathrm{V}_{\varphi}}{\mathrm{R}}\right]=\mathrm{F}_{\varphi} \mathrm{R}
$$

Similarly, for $\mathrm{q}_{\mathrm{i}}=\mathrm{z}$, we have 
Mechanical Engineering: An International Journal (MEIJ), Vol. 4, No. 1/2/3/4, November 2017

$$
\begin{aligned}
& \rho\left(\frac{\mathrm{D} \dot{\mathrm{z}}}{\mathbf{D t}}\right)=\mathrm{Q}_{\mathrm{z}}=\mathrm{F}_{\mathrm{z}} \\
& \rho \frac{\mathrm{DV} \mathrm{z}}{\mathrm{Dt}}=-\frac{\partial \mathrm{p}}{\partial \mathrm{z}}
\end{aligned}
$$

Where $\mathrm{V}_{\mathrm{R}}=\dot{\mathrm{R}}, \mathrm{V}_{\varphi}=\mathrm{R} \dot{\varphi}, \mathrm{V}_{\mathrm{z}}=\dot{\mathrm{z} a n d}$

$$
\frac{\mathrm{D}}{\mathrm{Dt}}=\frac{\partial}{\partial \mathrm{t}}+\mathrm{V}_{\mathrm{R}} \frac{\partial}{\partial \mathrm{R}}+\frac{\mathrm{V}_{\varphi}}{\mathrm{R}} \frac{\partial}{\partial \varphi}+\mathrm{V}_{z} \frac{\partial}{\partial z}
$$

\section{SPHerical CoORdinateS}

$$
\begin{aligned}
& \mathrm{d} \mathbf{R}=\mathrm{dr} \mathbf{e}_{\mathrm{r}}+\mathrm{r} d \theta \mathbf{e}_{\theta}+\mathrm{r} \sin \theta \mathrm{d} \varphi \mathbf{e}_{\varphi} \\
& \mathbf{V}=\dot{\mathrm{r}} \mathbf{e}_{\mathrm{r}}+\mathrm{r} \dot{\theta} \mathbf{e}_{\theta}+\mathrm{r} \dot{\varphi} \sin \theta \mathbf{e}_{\varphi} \\
& \mathrm{T}=\frac{1}{2} \mathrm{~V}^{2}=\frac{1}{2}\left[\dot{\mathrm{r}}^{2}+(\mathrm{r} \dot{\theta})^{2}+(\mathrm{r} \dot{\varphi} \sin \theta)^{2}\right] \\
& \frac{\partial \mathbf{R}}{\partial \mathrm{r}}=\mathbf{e}_{\mathrm{r}} \quad \frac{\partial \mathrm{R}}{\partial \theta}=\mathrm{r} \mathbf{e}_{\theta} \quad \frac{\partial \mathrm{R}}{\partial \varphi}=\mathrm{r} \sin \theta \mathbf{e}_{\varphi}
\end{aligned}
$$

For $\mathrm{q}_{\mathrm{i}}=\mathrm{r}$, we have

$$
\frac{\partial \mathrm{T}}{\partial \dot{\mathrm{r}}}=\dot{\mathrm{r}} \quad \frac{\partial \mathrm{T}}{\partial \mathrm{r}}=\mathrm{r} \dot{\theta}^{2}+\mathrm{r} \dot{\varphi}^{2}(\sin \theta)^{2}
$$

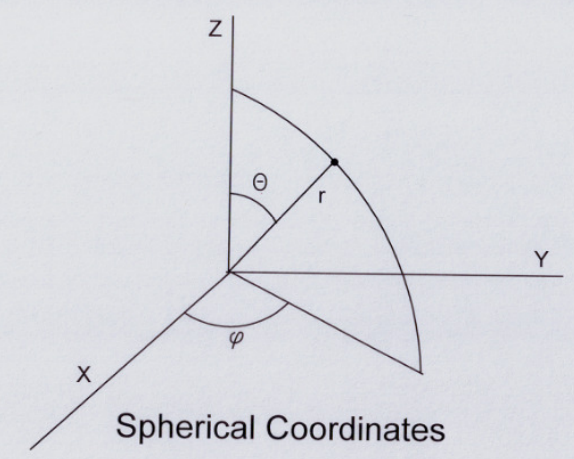

$$
\begin{aligned}
& \rho\left\{\frac{\mathrm{D} \dot{\mathrm{r}}}{\mathrm{Dt}}-\left[\mathrm{r} \dot{\theta}^{2}+\mathrm{r}(\dot{\varphi} \sin \theta)^{2}\right]\right\}=\mathrm{Q}_{\mathrm{r}} \\
& \rho\left[\frac{\mathrm{D} \mathrm{V}_{\mathrm{r}}}{\mathrm{Dt}}-\frac{1}{\mathrm{r}}\left(\mathrm{V}_{\theta}^{2}+\mathrm{V}_{\varphi}^{2}\right)\right]=\mathrm{Q}_{\mathrm{r}}=-\frac{\partial \mathrm{p}}{\partial \mathrm{r}}+\rho \mathbf{g} \cdot \mathbf{e}_{\mathrm{r}}
\end{aligned}
$$

For $\mathrm{q}_{i}=\theta$, we have 
Mechanical Engineering: An International Journal (MEIJ), Vol. 4, No. 1/2/3/4, November 2017

$$
\begin{gathered}
\frac{\partial \mathrm{T}}{\partial \dot{\theta}}=\mathrm{r}^{2} \dot{\theta} \quad \frac{\partial \mathrm{T}}{\partial \theta}=\mathrm{r}^{2} \dot{\varphi}^{2} \sin \theta \cos \theta \\
\rho\left[\frac{\mathrm{D}}{\mathrm{Dt}}\left(\mathrm{r}^{2} \dot{\theta}\right)-\mathrm{r}^{2} \dot{\varphi}^{2} \sin \theta \cos \theta\right]=\mathrm{Q}_{\theta} \\
\rho\left[\frac{\mathrm{D}}{\mathrm{Dt}}\left(\mathrm{r} \mathrm{V}_{\theta}\right)-\mathrm{V}_{\varphi}^{2} \cot \theta\right]=\mathrm{F}_{\theta} \mathrm{r} \\
\rho \mathrm{r} \frac{\mathrm{DV} \theta}{\mathrm{Dt}}+\rho \mathrm{V}_{\theta} \mathrm{V}_{\mathrm{r}}-\rho \mathrm{V}_{\varphi}^{2} \cot \theta=\mathrm{F}_{\theta} \mathrm{r} \\
\rho \frac{\mathrm{DV} \theta}{\mathrm{Dt}}+\rho \frac{\mathrm{V}_{\theta} \mathrm{V}_{\mathrm{r}}}{\mathrm{r}}-\rho \frac{\mathrm{V}_{\varphi}^{2}}{\mathrm{r}} \cot \theta=-\frac{1}{\mathrm{r}} \frac{\partial \mathrm{p}}{\partial \theta}+\rho \mathbf{g} \cdot \mathbf{e}_{\theta}
\end{gathered}
$$

Similarly for $\mathrm{q}_{i}=\varphi$, we have

$$
\begin{aligned}
& \rho \frac{\mathrm{D}}{\mathrm{Dt}}\left[(\mathrm{r} \sin \theta)^{2} \dot{\varphi}\right]=Q_{\varphi}=\mathrm{F} \cdot \frac{\partial \mathrm{R}}{\partial \varphi}=\mathrm{F} \cdot \mathrm{r} \sin \theta \mathbf{e}_{\varphi}=\mathrm{F}_{\varphi} \mathrm{r} \sin \theta \\
& \rho \frac{\mathrm{D}}{\mathrm{Dt}}\left(\mathrm{r} \sin \theta \mathrm{V}_{\varphi}\right)=\rho \mathrm{r} \sin \theta \frac{\mathrm{DV} \mathrm{V}_{\varphi}}{\mathrm{Dt}}+\rho \mathrm{V}_{\mathrm{r}} \sin \theta \mathrm{V}_{\varphi}+\rho \mathrm{r} \cos \theta \dot{\theta} \mathrm{V}_{\varphi}=\mathrm{F}_{\varphi} \mathrm{r} \sin \theta \\
& \rho \frac{\mathrm{DV}}{\mathrm{Dt}}+\rho \frac{\mathrm{V}_{\mathrm{r}} \mathrm{V}_{\varphi}}{\mathrm{r}}+\rho \frac{\mathrm{V}_{\varphi} \mathrm{V}_{\theta}}{\mathrm{r}} \cot \theta=\mathrm{F}_{\varphi}=-\frac{1}{\mathrm{r} \sin \theta} \frac{\partial \mathrm{p}}{\partial \varphi}+\rho \mathbf{g} \cdot \mathbf{e}_{\varphi}
\end{aligned}
$$

whereV $\mathrm{r}_{\mathrm{r}}=\dot{\mathrm{r}}, \mathrm{V}_{\theta}=\mathrm{r} \dot{\theta}, \mathrm{V}_{\varphi}=\mathrm{r} \sin \theta \dot{\varphi}$

$$
\frac{\mathrm{D}}{\mathrm{Dt}}=\frac{\partial}{\partial \mathrm{t}}+\mathrm{V}_{\mathrm{r}} \frac{\partial}{\partial \mathrm{r}}+\frac{\mathrm{V}_{\theta}}{r} \frac{\partial}{\partial \theta}+\frac{\mathrm{V}_{\varphi}}{\mathrm{r} \sin \theta} \frac{\partial}{\partial \varphi}
$$

\section{ConClusion}

This paper may be considered as the first step for the use of Lagrangian equation in fluid mechanics. An inviscid incompressible fluid is considered here, more general form of the Lagrangian equation can be developed for more general kind of fluid. Now the door is open, further development and application of the equation can be very fruitful to scientists in fluid mechanics.

\section{REFERENCE}

1. Ying, S. J. ‘ Advanced Dynamics ‘ AIAA Education Series 1997. 